\title{
Fortalezas y dificultades para el ajuste emocional en niños aymara desde la perspectiva de los menores, padres y profesores
}

\author{
A. Caqueo • A. Urzúa ・ R. Ferrer・F. Zúñiga • C. Palma •J. Escudero
}

\begin{abstract}
Strengths and difficulties in emotional adjustment of Aymara children from the perspective of children, parents and teachers

Objective: To describe capabilities and difficulties in emotional adjustment experienced by children living in the Chilean Aymara city of Arica. Patients and Method: 610 students between 5th and 8th grade, in addition to their parents and teachers were surveyed using the Strengths and Difficulties Questionnaire (SDQ), comparing Aymara children to those without indigenous heritage. 25 items divided into five scales were evaluated: Emotional, behavioral problems, hyperactivity, relationship problems with peers and prosocial behaviors, classifying the respondents into three levels, Normal, Border and Abnormal. Results: There were no significant differences among the groups studied, where discrepancies in almost all cases show a slight effect $(d \geq|0.2|)$. Also, no relevant effects were observed regarding the type of assessor on the assessment of each dimension. Despite this, it was observed that Aymara children showed lower scores than their peers in "behavioral problems" according to their teachers, but this difference was also mild (partial $\eta^{2}>0.01$ ). Conclusions: Aymara Children showed similar strengths and difficulties than non-Aymara students in situations that require emotional adjustment.
\end{abstract}

(Key words: Ethnic groups, emotional aspects, Aymara, etnia, indigenous).

Rev Chil Pediatr 2014; 85 (5): 561-568

\section{RESUMEN}

Objetivo: Describir capacidades y dificultades en el ajuste emocional experimentadas por niños aymara residentes en la ciudad chilena de Arica. Pacientes y Método: Se encuestó a 610 alumnos de $5^{\circ}$ a $8^{\circ}$ año de En-

Recibido el 30 de enero de 2014, última versión aceptada para publicación el 13 de septiembre de 2014.

\section{Alejandra Caqueo-Uríar $(\bowtie)$}

Doctora en Psicología Clínica y de la Salud. Universidad de Tarapacá.

E-mail: acaqueo@uta.cl

Alfonso Urzúa M.

Doctor en Psicología Clínica y de la Salud. Universidad Católica del Norte.

Rodrigo Ferrer U.

Doctor en Metodología de las Ciencias Sociales y de la Salud. Universidad de Tarapacá.

Francisco Zúñiga M., Cristián Palma F., Jorge Escudero B.

Psicólogo, Licenciado en Psicología. Universidad de Tarapacá. 
señanza General Básica de la ciudad de Arica, además de sus padres y profesores, mediante el Cuestionario de Capacidades y Dificultades (SDQ), comparando a niños aymara con aquellos sin ascendencia indígena. Se evaluaron 25 ítems divididos en 5 escalas: emocional, problemas de comportamiento, hiperactividad, problemas de relación con pares y conductas prosociales, clasificando a los encuestados en tres niveles, Normal, Límite o Anormal. Resultados: No hubo diferencias poblacionales relevantes en las dimensiones del SDQ entre grupos estudiados, donde las discrepancias en prácticamente todos los casos muestran un efecto leve $(d \geq|0,2|)$. Tampoco se observaron efectos poblacionales relevantes respecto del tipo de evaluador sobre la valoración de cada dimensión. Pese a esto, cabe señalar que niños aymara mostraron una menor puntuación que sus pares en "problemas de comportamiento" según sus profesores, pero dicha interacción poblacional también fue leve $\left(\eta^{2}\right.$ parcial $\left.>0,01\right)$. Conclusiones: Niños aymara mostraron fortalezas y dificultades similares a las de sus pares no aymara ante situaciones que requieren ajuste emocional.

(Palabras clave: Grupos étnicos, aspectos emocionales, Aymara, etnia, indigena).

Rev Chil Pediatr 2014; 85 (5): 561-568

\section{Introducción}

La comunidad aymara, ubicada principalmente en la pre-cordillera andina de la región de Arica y Parinacota, ha vivido un proceso migratorio desde sus poblados rurales hacia la ciudad de Arica, lo cual ha significado una de las experiencias más complejas que ha tenido que enfrentar ${ }^{1}$. A partir de dicho proceso se producen zonas de contacto entre las diferentes prácticas o ámbitos culturales ${ }^{2}$.

Existe características particulares que pudieran influir en el ajuste emocional de los niños aymara que emigran a ciudades. Ellos reciben de la familia y parientes una parte sustantiva de los valores que dirigirán su conducta y desempeño en las distintas fases de su vida, proyecto de socialización definido como "enseñar a vivir bien" (suma q'maña), reportando un menor riesgo de Trastorno por Déficit Atencional con Hiperactividad (TDAH), atribuido a las estrictas normas ancestrales reforzadas por la comunidad ${ }^{3}$. Desde la perspectiva socioeconómica, los aymara evidencian una mayor pobreza respecto al promedio nacional, e incluso levemente superior al promedio exhibido por los pueblos indígenas de Chile 4 . Junto a eso, aquellos niños que han emigrado con sus familias a ciudades de la pampa o de la costa, van adaptándose tímidamente a la vida urbana, sufriendo discriminaciones por su fenotipo andino 5 .

De este modo, niños y adolescentes pueden presentar problemas y dificultades al enfrentar situaciones que requieren adaptación, con tasas de prevalencia de trastornos mentales entre el 5 y el 20\%, según los criterios diagnósticos (DSM-IV; CIE-10), ocasionando un fuerte impacto tanto en las esferas personal, académica, familiar y social, como a nivel económico y sanitario $0^{6,7}$. Cerca de un $50 \%$ de los trastornos mentales comienzan antes de los 15 años y, en muchos casos, dicha sintomatología permanece hasta la edad adulta, variando en función del sexo y la edad de los adolescentes ${ }^{7-9}$. Nuestro objetivo fue describir las capacidades y dificultades del ajuste emocional de menores emigrantes aymara, residentes en la ciudad de Arica.

\section{Pacientes y Método}

\section{Participantes}

Alumnos de $5^{\circ}$ a $8^{\circ}$ año de Enseñanza General Básica, pertenecientes a nueve Establecimientos Educacionales de la ciudad de Arica. Cuatro de ellos corresponden a Escuelas Públicas y cinco a Colegios Privados Subvencionados por el Estado. Los establecimiento fueron seleccionados en un esfuerzo por maximizar el número de aymara incluido en el estudio. Todos pertenecían a un nivel socioeconómico bajo.

\section{Instrumentos}

Cuestionario de Capacidades y Dificultades (SDQ) de Goodman, 1997: Este instru- 
mento se utiliza para evaluación de problemas comportamentales en niños de 3 a 16 años. Las propiedades psicométricas muestran una adecuado nivel de confiabilidad y validez, con Alpha de Cronbach =0,73 (10). Cuenta con 25 ítems divididos en 5 escalas: "emocional", "problemas de comportamiento", "hiperactividad", "problemas de relación con pares" y "conductas prosociales". La puntuación total de dificultades se genera mediante la adición de las puntuaciones de las cuatro primeras escalas. Hay tres versiones del cuestionario: padres, profesores y auto-informe. Con las puntuaciones obtenidas, los evaluados son catalogados tres niveles diagnósticos: Normal, Límite o Anormal.

\section{Datos socio-demográficos familiares}

Se elaboraron dos fichas, una respondida por los apoderados y otra por los niños, en una versión modificada y con lenguaje sencillo.

\section{Procedimiento}

Se realizó un catastro de Escuelas Municipales y Colegios Particulares Subvencionados que tuvieran un número relevante de alumnos(as) de etnia aymara, según listado de beneficiarios de los años 2010-2011 de la Beca Indígena, otorgada por la Junta Nacional de Auxilio Escolar y Becas del Ministerio de Educación. Una vez seleccionados los establecimientos se les invitó a participar en el estudio bajo autorización de sus educadores, consentimiento informado de sus padres y asentimiento por parte de los niños. La evaluación se realizó en el colegio en horario de clase. Los criterios de inclusión para el grupo de niños y niñas aymara fueron que el menor tuviera al menos un apellido aymara establecido en la Ley Indígena, y la autoadscripción a dicha etnia, o sea, el reconocimiento como indígena tanto de los padres como del propio niño(a). Niños que presentaron ascendencia indígena distinta a la aymara fueron excluidos del estudio.

\section{Análisis de datos}

Para valorar diferencias poblacionales en las puntuaciones del SDQ asociadas a la etnia, se procedió a realizar una prueba $t$ de Student para muestras independientes. $d$ de Cohen fue utilizada para medir el tamaño del efecto, cuyos valores entre $0,2-0,5$ indican efectos leves, $0,5-0,8$ efectos moderados y $>0,8$ efectos altos. Para valorar discrepancias entre las valoraciones de cada dimensión dependiendo del tipo de evaluador y si estas se encuentran relacionadas con la etnia del niño, se utilizó una ANOVA de medidas repetidas; valores de $\eta^{2}$ parcial entre 0,01-0,06 indican efectos leves, 0,06-0,14 efectos moderados y $>0,14$ efectos altos. Para analizar el comportamiento de los efectos principales detectados se realizaron pruebas post-hoc para medias repetidas con ajuste Bonferroni. Para valorar el poder predictivo sobre el rendimiento escolar de las cinco dimensiones del SDQ por cada uno de los evaluadores, se realizó un análisis de regresión lineal mediante el método de pasos sucesivos. El estadístico de Durbin-Watson fue utilizado para probar la relación de los residuos de las variables independientes en el análisis de regresión; valores entre 1,5-2,5 permiten asumir el supuesto de independencia. El estadístico de tolerancia fue empleado para evaluar el supuesto de no colinealidad, considerando que valores $<0,1$ indican problemas de colinealidad. El factor de inflación de la varianza (FIV) fue usado para probar el supuesto de no colinealidad, donde valores $>10$ indican problemas de colinealidad. La información fue analizada usando SPSS en su versión 21.

\section{Resultados}

Un total de 306 niños $(50,1 \%)$ y 304 niñas $(49,9 \%)$ fueron evaluados, 246 aymaras $(40,3 \%)$ y 364 sin ascendencia indígena detectable $(59,7 \%)$, cuya edad fue de $11,89 \pm 1,45$ años. No se observa ninguna asociación entre sexo y etnia, por lo cual puede mantenerse la hipótesis nula de independencia y asumir que la etnicidad se distribuye indistintamente entre niños y niñas $\left(\chi_{\mathrm{GL}=1}^{2}=0,53 ; \mathrm{p}=0,47\right)$.

De las 5 dimensiones evaluadas por cada uno de los evaluadores, sólo tres presentaron diferencias poblacionales, una de ellas concordante entre padres y niños. Las diferencias en prácticamente todo los casos corresponden 
a un efecto leve $(d \geq|0,2|)$, con la salvedad de la evaluación que los padres hacen de conductas prosociales, en la cual, pese a presentar diferencias significativas, puede suponerse una diferencia poblacional irrelevante $(d<|0,2|)$ (tabla 1).

En todas las dimensiones evaluadas se observan efectos poblacionales del tipo de evaluador. El tamaño del efecto de dicha influencia es leve para las dimensiones "problemas de relación con los pares", "conductas prosociales" e "hiperactividad"; y alta para "emocional" y "problemas de comportamiento". En lo que respecta a los efectos de interacción, sólo se observan efectos de interacción poblacional del tipo de evaluador con la etnia del niño en la dimensión "problemas de comportamiento", aunque esta asociación tiene un efecto leve (tabla 2).

Respecto a la dimensión "conductas prosociales", se observa que son los padres los que asignan mayor puntaje a los niños en esta di- mensión, seguido de profesores y niños. En la dimensión "emocional" son los padres quienes asignan mayores puntajes, seguido por los niños y profesores. En la dimensión "problemas de comportamiento" los padres asignan puntuaciones ostensiblemente mayores que los niños, siendo los profesores quienes asignan puntuaciones menores. En la dimensión "hiperactividad" los profesores asignan puntuaciones menores a las que asignan padres y niños. Finalmente, en la dimensión "problemas de relación con pares", los profesores asignan puntuaciones menores a padres y niños (tabla 3 ).

Respecto al efecto de la interacción de la dimensión "problemas de comportamiento", se encontró sólo una leve variación asociada a la etnia cuando la valoración es realizada por los profesores. En el grupo no aymara se observa que, a partir de un modelo de 5 variables, se reducen los errores de predicción sobre el rendimiento académico en un $36 \%$, no observándose violaciones al supuesto de inde-

Tabla 1. Diferencias de media en las puntuaciones del SDQ según tipo de evaluador y origen étnico

\begin{tabular}{|llllllccc|}
\hline Evaluador & Dimensión & $\mathbf{t}$ & $\mathbf{G L}$ & $\mathbf{p}$ & $\begin{array}{c}\text { Diferencia de } \\
\text { medias (aymara- } \\
\text { No aymara) }\end{array}$ & $\begin{array}{c}\text { Intervalo de } \\
\text { confianza (95\%) } \\
\text { Inferior }\end{array}$ Superior & $\begin{array}{c}\text { d de } \\
\text { Cohen }\end{array}$ \\
Niños & Problemas de relación con pares & 3,29 & 607 & 0,001 & 0,43 & 0,17 & 0,69 & 0,27 \\
Padres & Conductas prosociales & $-2,01$ & 607 & 0,045 & $-0,29$ & $-0,58$ & $-0,01$ & $-0,17$ \\
& Problemas de relación con pares & 3,25 & 603 & 0,001 & 0,45 & 0,18 & 0,73 & 0,27 \\
Profesores & Problemas de comportamiento* & $-3,13$ & 567,30 & 0,002 & $-0,42$ & $-0,68$ & $-0,16$ & $-0,26$ \\
\hline
\end{tabular}

*No asumiendo igualdad de varianza. GL: grados de libertad.

Tabla 2. Efectos principales del tipo de evaluador, y su interacción con la etnia del niño, para cada una de las dimensiones

\begin{tabular}{|c|c|c|c|c|c|c|}
\hline Dimensión & Efecto & $\begin{array}{c}\text { Media } \\
\text { cuadrática }\end{array}$ & $\mathbf{F}$ & GL & $\mathbf{p}$ & $\eta^{2}$ parcial \\
\hline \multirow[t]{2}{*}{ Conductas prosociales* } & Tipo de evaluador & 121,93 & 33,03 & 1,94 & 0,00 & 0,05 \\
\hline & Tipo de evaluador x etnia del niño & 3,44 & 0,93 & 1,94 & 0,39 & 0,00 \\
\hline \multirow[t]{2}{*}{ Emocional* } & Tipo de evaluador & 874,08 & 244,7 & 1,95 & 0,00 & 0,29 \\
\hline & Tipo de evaluador $x$ etnia del niño & 3,67 & 1,03 & 1,95 & 0,36 & 0,00 \\
\hline \multirow[t]{2}{*}{ Problemas de comportamiento } & Tipo de evaluador & 2690,84 & $1.274,8$ & 2 & 0,00 & 0,68 \\
\hline & Tipo de evaluador x etnia del niño & 7,45 & 3,53 & 2 & 0,03 & 0,01 \\
\hline \multirow[t]{2}{*}{ Hiperactividad* } & Tipo de evaluador & 102,06 & 33,01 & 1,90 & 0,00 & 0,05 \\
\hline & Tipo de evaluador $\mathrm{x}$ etnia del niño & 1,57 & 0,51 & 1,90 & 0,59 & 0,00 \\
\hline \multirow[t]{2}{*}{ Problemas de relación con pares } & Tipo de evaluador & 43,51 & 20,0 & 2 & 0,00 & 0,03 \\
\hline & Tipo de evaluador $x$ etnia del niño & 5,94 & 2,73 & 2 & 0,07 & 0,01 \\
\hline
\end{tabular}

*No asumiendo esfericidad. GL: grados de libertad. 
Tabla 3. Comparaciones post-hoc de los efectos del tipo de evaluador para cada dimensión

\begin{tabular}{|c|c|c|c|c|c|c|c|}
\hline \multirow[t]{2}{*}{ Dimensión } & \multirow{2}{*}{\multicolumn{2}{|c|}{ Tipo de evaluador }} & \multirow{2}{*}{$\begin{array}{c}\text { Diferencia } \\
\text { de medias } \\
(I-J)\end{array}$} & \multirow{2}{*}{$\begin{array}{c}\text { Error típico } \\
\text { de la } \\
\text { diferencia }\end{array}$} & \multirow[t]{2}{*}{$\mathbf{p}$} & \multicolumn{2}{|c|}{$\begin{array}{l}\text { Intervalo de confianza a } \\
\mathbf{9 5 \%} \text { para la diferencia }\end{array}$} \\
\hline & & & & & & $\begin{array}{l}\text { Límite } \\
\text { inferior }\end{array}$ & $\begin{array}{l}\text { Límite } \\
\text { superior }\end{array}$ \\
\hline $\begin{array}{l}\text { Conductas } \\
\text { prosociales }\end{array}$ & $\begin{array}{l}\text { Niños (I) } \\
\text { Padres (I) }\end{array}$ & $\begin{array}{l}\text { Padres }(J) \\
\text { Profesores }(J) \\
\text { Niños }(J) \\
\text { Profesores }(J)\end{array}$ & $\begin{array}{r}-0,89 \\
-0,58 \\
0,89 \\
0,31\end{array}$ & $\begin{array}{l}0,10 \\
0,12 \\
0,10 \\
0,11\end{array}$ & $\begin{array}{l}0,00 \\
0,00 \\
0,00 \\
0,02\end{array}$ & $\begin{array}{r}-1,13 \\
-0,86 \\
0,64 \\
0,04\end{array}$ & $\begin{array}{r}-0,64 \\
-0,29 \\
1,13 \\
0,58\end{array}$ \\
\hline Emocional & $\begin{array}{l}\text { Niños (I) } \\
\text { Padres (I) }\end{array}$ & $\begin{array}{l}\text { Padres }(J) \\
\text { Profesores }(J) \\
\text { Niños }(J) \\
\text { Profesores }(J)\end{array}$ & $\begin{array}{r}-0,77 \\
1,60 \\
0,77 \\
2,36\end{array}$ & $\begin{array}{l}0,10 \\
0,12 \\
0,10 \\
0,11\end{array}$ & $\begin{array}{l}0,00 \\
0,00 \\
0,00 \\
0,00\end{array}$ & $\begin{array}{r}-1,01 \\
1,32 \\
0,52 \\
2,11\end{array}$ & $\begin{array}{r}-0,52 \\
1,88 \\
1,01 \\
2,62\end{array}$ \\
\hline $\begin{array}{l}\text { Problemas de } \\
\text { comportamiento }\end{array}$ & $\begin{array}{l}\text { Niños (I) } \\
\text { Padres (I) }\end{array}$ & $\begin{array}{l}\text { Padres }(J) \\
\text { Profesores }(J) \\
\text { Niños }(J) \\
\text { Profesores }(J)\end{array}$ & $\begin{array}{r}-2,98 \\
1,19 \\
2,98 \\
4,17\end{array}$ & $\begin{array}{l}0,08 \\
0,09 \\
0,08 \\
0,09\end{array}$ & $\begin{array}{l}0,00 \\
0,00 \\
0,00 \\
0,00\end{array}$ & $\begin{array}{r}-3,18 \\
0,98 \\
2,78 \\
3,97\end{array}$ & $\begin{array}{r}-2,78 \\
1,40 \\
3,18 \\
4,37\end{array}$ \\
\hline Hiperactividad & $\begin{array}{l}\text { Niños (I) } \\
\text { Padres (I) }\end{array}$ & $\begin{array}{l}\text { Padres }(J) \\
\text { Profesores (J) } \\
\text { Niños }(J) \\
\text { Profesores }(J)\end{array}$ & $\begin{array}{r}0,02 \\
0,72 \\
-0,02 \\
0,70\end{array}$ & $\begin{array}{l}0,09 \\
0,11 \\
0,09 \\
0,10\end{array}$ & $\begin{array}{l}1,00 \\
0,00 \\
1,00 \\
0,00\end{array}$ & $\begin{array}{r}-0,19 \\
0,46 \\
-0,23 \\
0,45\end{array}$ & $\begin{array}{l}0,23 \\
0,97 \\
0,19 \\
0,94\end{array}$ \\
\hline $\begin{array}{l}\text { Problemas de } \\
\text { relación con pares }\end{array}$ & $\begin{array}{l}\text { Niños (I) } \\
\text { Padres (I) }\end{array}$ & $\begin{array}{l}\text { Padres }(\mathrm{J}) \\
\text { Profesores }(\mathrm{J}) \\
\text { Niños }(\mathrm{J}) \\
\text { Profesores }(\mathrm{J})\end{array}$ & $\begin{array}{r}-0,14 \\
0,39 \\
0,14 \\
0,53\end{array}$ & $\begin{array}{l}0,08 \\
0,09 \\
0,08 \\
0,09\end{array}$ & $\begin{array}{l}0,30 \\
0,00 \\
0,30 \\
0,00\end{array}$ & $\begin{array}{r}-0,34 \\
0,18 \\
-0,06 \\
0,31\end{array}$ & $\begin{array}{l}0,06 \\
0,60 \\
0,34 \\
0,74\end{array}$ \\
\hline
\end{tabular}

Tabla 4. Modelo de regresión lineal para el grupo no aymara

\begin{tabular}{|c|c|c|c|c|c|c|}
\hline $\mathbf{R}^{2}$ & $\mathbf{R}_{\text {corregido }}^{2}$ & $\begin{array}{l}\text { Error típico de } \\
\text { estimación }\end{array}$ & $\begin{array}{c}\text { Media } \\
\text { cuadrática }\end{array}$ & $\mathbf{F}_{\mathrm{GL}=\mathbf{5}}$ & $\mathbf{p}$ & $\begin{array}{c}\text { Estadístico } \\
\text { de Durbin- } \\
\text { Watson }\end{array}$ \\
\hline 0,37 & 0,36 & 0,46 & 7,16 & 39,23 & 0,00 & 2,00 \\
\hline $\begin{array}{l}\text { Tipo de } \\
\text { evaluador }\end{array}$ & Dimensión & $\mathbf{b}_{\text {tipificado }}$ & $t$ & $p$ & $\begin{array}{l}\text { Estadístico } \\
\text { de tolerancia }\end{array}$ & FIV \\
\hline Niños & Hiperactividad & $-0,17$ & $-3,74$ & 0,00 & 0,94 & 1,07 \\
\hline Padres & Emocional & $-0,12$ & $-2,75$ & 0,00 & 0,97 & 1,03 \\
\hline Profesores & $\begin{array}{l}\text { Hiperactividad } \\
\text { Conductas prosociales } \\
\text { Emocional }\end{array}$ & $\begin{array}{l}-0,35 \\
0,20 \\
-0,11\end{array}$ & $\begin{array}{l}-7,15 \\
4,28 \\
-2,34\end{array}$ & $\begin{array}{l}0,00 \\
0,00 \\
0,02\end{array}$ & $\begin{array}{l}0,78 \\
0,84 \\
0,82\end{array}$ & $\begin{array}{l}1,28 \\
1,19 \\
1,22\end{array}$ \\
\hline
\end{tabular}

pendencia $(1,5<D W<2,5)$, ni al supuesto de no colinealidad $(E T>10$ y $F I V<10)$. Cuando estas variables se consideraron en conjunto, la valoración que los profesores realizan de la dimensión "hiperactividad" predice una reducción en el promedio de notas de un $12 \%$, respecto al pronóstico del promedio por cada punto que se incrementa la escala; la valoración de la dimensión "conductas prosociales" predice un incremento de un $4 \%$, y la valoración de la dimensión "emocional" predice una disminución de un 1\%. En el caso de los niños, sólo la valoración de la dimensión "hiperactividad" presenta un efecto no nulo en la población, prediciendo una reducción en el promedio de notas de un 3\% respecto al promedio. Finalmente, en el caso de la evaluación de los padres, sólo la dimensión "emocional" presen- 
Tabla 5. Modelo de regresión lineal para el grupo aymara

\begin{tabular}{|c|c|c|c|c|c|c|}
\hline $\mathbf{R}^{2}$ & $\mathbf{R}_{\text {corregido }}^{2}$ & $\begin{array}{l}\text { Error típico de } \\
\text { estimación }\end{array}$ & $\begin{array}{c}\text { Media cuadrá- } \\
\text { tica }\end{array}$ & $\mathbf{F}_{\mathrm{GL}=3}$ & $\mathbf{p}$ & $\begin{array}{c}\text { Estadístico } \\
\text { de Durbin- } \\
\text { Watson }\end{array}$ \\
\hline 0,23 & 0,22 & 0,49 & 5,59 & 23,06 & 0,00 & 1,94 \\
\hline $\begin{array}{l}\text { Tipo de } \\
\text { evaluador }\end{array}$ & Dimensión & $\mathbf{b}_{\text {tipificado }}$ & $\mathbf{T}$ & $\mathbf{p}$ & $\begin{array}{l}\text { Estadístico } \\
\text { de tolerancia }\end{array}$ & FIV \\
\hline \multirow[t]{3}{*}{ Profesores } & Hiperactividad & $-0,31$ & $-5,21$ & 0,00 & 0,92 & 1,09 \\
\hline & Conductas prosociales & 0,18 & 2,90 & 0,00 & 0,81 & 1,24 \\
\hline & $\begin{array}{l}\text { Problemas de relación } \\
\text { con pares }\end{array}$ & $-0,16$ & $-2,47$ & 0,01 & 0,83 & 1,21 \\
\hline
\end{tabular}

ta un efecto significativo, prediciendo una leve reducción en el promedio de notas de un $1 \%$ respecto al promedio (tabla 4 ).

En el caso del grupo aymara se observa que, a partir de un modelo de 3 variables se logra reducir los errores de predicción sobre el rendimiento académico en un $22 \%$, no observándose violaciones al supuesto de independencia ni al supuesto de no colinealidad. Sólo la valoración que realizaron los profesores presentó un efecto no nulo en la población. La dimensión "hiperactividad" predijo una reducción en el promedio de notas de un $10 \%$, respecto al pronóstico de la media, por cada punto que se incrementa la escala; la dimensión "conductas prosociales" predijo un incremento de un 3\%; $\mathrm{y}$, finalmente, la valoración de la dimensión "problemas de relación con pares" predijo una disminución de un 3\% (tabla 5).

\section{Discusión}

Si bien se observan diferencias poblacionales respecto de las capacidades y dificultades al comparar a los niños aymara con los no aymara, éstas se califican como leves desde un aspecto metodológico cuantitativo. En general, las capacidades y dificultades que muestran ambos grupos suelen ser homogéneas cuando se utiliza como fuente de información el reporte de profesores, padres y el autoreporte de niños(as). Esto puede explicarse por el fenómeno de occidentalización, influyendo en la adaptación de niños aymara a la cultura dominante, logrando desplegar fortalezas ante situaciones problemáticas de modo muy similar al de sus pares ${ }^{11}$.
Existen discrepancias en la percepción que padres, profesores y niños reportan respecto de las capacidades y dificultades evaluadas en ambos grupos, en relación a los "problemas de comportamiento" y "emociones". Al evaluar si la pertenencia a la etnia aymara influye en ambas dimensiones, sólo la percepción de los "problemas de comportamiento" tuvo una leve influencia, no explicando las variaciones en el reporte entregado. Es decir, el pertenecer a la etnia aymara no incide mayormente en presentar problemas de conducta.

Los profesores suelen observan un menor número de problemas comportamentales en los niños aymara en comparación con los no aymara. Este hallazgo podría asociarse a los procesos de aculturación que niños y niñas aymara establecen con sus pares a la vez que se producen zonas de contacto entre diferentes prácticas culturales, donde se consideran diferentes marcos normativos que influyen en las conductas tanto a nivel escolar como familiar ${ }^{2}$. Sin embargo, estudios previos han señalado que los niños aymara tienden a ser más ordenados, ponen mayor atención en clases y no molestan a sus compañeros, reafirmándose en esta investigación dichos resultados ${ }^{3}$.

Realizando un análisis de la muestra general, los padres suelen observar mayores conductas prosociales en sus hijos(as), señalando mayores problemas emocionales y de comportamiento en comparación con la observación de profesores y niños. Por su parte, los profesores observan un bajo nivel de conductas hiperactivas y problemas de relación con pares en comparación con el reporte de los padres.

Respecto al reporte de los profesores de 
los niños no aymara, la presencia de conductas hiperactivas y problemas emocionales que podrían manifestarse a través de quejas somáticas suele predecir una disminución en el rendimiento académico. Se hace relevante pesquisar mayores antecedentes que apunten a mejorar la precisión de la observación respecto de ellas, a fin de prevenir eventuales dificultades académicas vinculadas a un TDAH, dado que este último surge en la infancia y se caracteriza por un patrón persistente de inatención, hiperactividad e impulsividad que incide negativamente en el desempeño académico, cognoscitivo y social de quien lo padece ${ }^{12}$. Cabe señalar que, al igual que sus profesores, los niños no aymara informan que las conductas hiperactivas se asocian a una reducción de su rendimiento académico, apreciando que una carencia de concentración, inquietud psicomotora y bajo control conductual interfieren en la posibilidad de alcanzar un desempeño académico satisfactorio.

En relación a los problemas emocionales, resulta importante prestar atención a dicha variable, dado que estas dificultades ocurren con mayor frecuencia en aquellos niños que viven en condiciones de adversidad familiar ${ }^{13}$. A modo de visualización de capacidades y recursos protectores, la presencia de conductas prosociales suelen vincularse a un aumento del rendimiento académico de los niños no aymara. Estas conductas involucran toda acción social positiva que se realiza para beneficiar a otro con o sin motivación altruista ${ }^{14}$.

En el reporte de los profesores de conductas hiperactivas, se observa que la presencia de este tipo sintomatología suele ser bajas en niños y niñas aymara, en concordancia con estudios previos ${ }^{3,15}$. A modo de recursos protectores, y del mismo modo que con los niños no aymara, la presencia de conductas prosociales prevé un aumento del rendimiento escolar, al mismo tiempo que disminuye la ocurrencia de problemas con pares, variable que suele mermar el normal desempeño académico en niños aymara.

Considerando que el objetivo de esta investigación fue describir las capacidades y dificultades presentes en niños y niñas aymara residentes en la ciudad de Arica, concluimos que éstos logran desplegar fortalezas y dificultades de modo similar a la de sus pares no aymara, ante situaciones que requieren de un ajuste emocional.

Pese a la importancia de los resultados obtenidos, la presente investigación presenta limitaciones: sólo se evaluó a niños aymara de Chile, por lo que los resultados no pueden ser generalizables a toda la población aymara infantil, sugiriendo además la necesidad incorporar en futuros estudios a niños de otras etnias, tanto dentro del país como mapuche, rapa nui o quechua, como fuera de éste. Si bien la muestra analizada no puede considerarse representativa del colectivo de niños aymara, el uso de una muestra probabilística y el tamaño de la misma, apoyan la extracción de conclusiones a partir del colectivo analizado. En segundo lugar, es probable que existan algunas dificultades cuando se utilizan conceptos psicológicos e instrumentos de medición pertenecientes a otras culturas ${ }^{16,17}$. A este respecto, cabe tener en cuenta, no obstante, que la comparación de nuestros resultados con los obtenidos por estudios de otros países con la misma metodología, parece apuntar a la validez de estos instrumentos. Finalmente, el estudio presenta un diseño transversal, por lo que sería importante realizar estudios longitudinales que permitan evaluar la consistencia de los hallazgos obtenidos a lo largo del tiempo ${ }^{18}$.

Con el fin de favorecer un adecuado desarrollo de los niños aymara y no aymara, se sugiere que tanto las familias como los establecimientos educacionales estimulen estrategias que promuevan conductas prosociales vinculadas a habilidades sociales, resolución de conflictos y expresión emocional, junto a la permanente vinculación con redes comunitarias de salud mental. Aquello apunta a una mejor calidad de vida de estos menores, pues se ha visto que el proceso de acomodación a una nueva cultura generaría pautas de comportamiento conducentes a ella ${ }^{19}$. Asimismo, futuras investigaciones deberían incluir la participación de un grupo de niños aymara con residencia rural, ya que en esta investigación sólo hubo residentes en la ciudad de Arica, quienes han habitado muchos años en el sector urbano $\mathrm{y}$ han vivido un proceso de aculturación a las 
normas locales dominantes ${ }^{20}$. Finalmente, futuros estudios podrían indagar respecto a los estilos educativos parentales desarrollados en familias aymara, dado que estas dinámicas tenderían a reducir los problemas comportamentales en los menores.

Agradecimientos: Esta investigación ha sido financiada por el Fondo Nacional de Desarrollo Científico y Tecnológico del Gobierno de Chile, FONDECYT, Proyecto N 1110472 y por el Convenio de Desempeño UTA-MINEDUC.

Potenciales conflictos de interés: Este trabajo cumple con los requisitos sobre consentimiento /asentimiento informado, comité de ética, financiamiento, estudios animales y sobre la ausencia de conflictos de intereses según corresponda.

\section{Referencias}

1.- Gundermann H: Las organizaciones étnicas y el discurso de la identidad en el norte de Chile. Estudios Atacameños. 2000; 19: 75-91.

2.- Temple D, Layme F, Michaux J, González M, Blanco E: Las estructuras elementales de la reciprocidad. TARI, Plural Editores 2003, Carreras de Antropología y Arqueología UMSA, La Paz.

3.- Rothhammer P, Carrasco X, Henríquez H, et al: Bajo riesgo de déficit atencional/hiperactividad en niños aymarás. Implicancias genéticas, antropológicas y culturales. Chungará. Revista de Antropología Chilena. 2005; 37: 145-9.

4.- Ministerio de Salud: Perfil epidemiológico básico de la población aymara del Servicio de Salud Arica, 2006. Descargado el 10 de septiembre de 2012. Disponible en: http://www.saludarica.cl/ind/perfil_epidemiologico.pdf

5.- Kirberg A: La salud del niño Aymara. Rev Chil Pediatr 2006; 77: 608-9.

6.- Blanchard LT, Gurka MJ, Blackman JA: Emotional, developmental, and behavioral health of American children and their families: a report from the 2003 National Survey of Children's Health. Pediatrics 2006; 117: 1202-12.

7.- Costello EJ, Mustillo S, Erkanli A, Keeler G, Angold A: Prevalence and development of psychiatric disorders in childhood and adolescence. Arch Gen Psychiatry 2003;
60: 837-44.

8.- Cohen P: Child development and personality disorder. Psychiatr Clin North Am 2008; 31: 477-93.

9.- Fonseca E, Paino M, Lemos S, Muñiz J: Prevalencia de la sintomatología emocional y comportamental en adolescentes españoles a través del Strengths and Dificulties Questionnaire (SDQ). Revista de Psicopatología y Psicología Clínica 2011; 16: 15-25.

10.- Goodman R: The Strengths and Difficulties Questionnaire: A research note. J Child Psychol Psychiatry. 1997; 38: 581-6.

11.- Villena $C$ : Estrategias de afrontamiento en niños Aymara escolarizados en la ciudad de Arica. (Tesis de grado). Universidad de Tarapacá, 2011.

12.- Calderón A: Trastorno por Déficit de Atención e Hiperactividad: Describiendo un trastorno de la Niñez, la adolescencia y la adultez. Bun Synapsis. 2011; 3; 24-7.

13.- Hankin B, Abramson L: Development of Gender Differences in Depression: An Elaborated Cognitive Vulnerability-Transactional Stress Theory. Psychol Bull 2010; 127: 773-96.

14.- Garaigordobil M: Intervención psicológica para desarrollar la personalidad infantil: Juego, conducta prosocial y creatividad. Madrid. Editorial Pirámide, 2003.

15.- Paz L, Silva C, Rothhammer P, et al: Riesgo de déficit atencional/hiperactividad en escolares Aymara RapaNui y de Santiago de Chile. Posible contribución de polimorfismos genéticos del sistema dopaminérgico. Rev Med Chile 2011; 139: 600-5.

16.- Bracken P, Giller J, Summerfield D: Psychological responses to war and atrocity: The limitations of the current concepts. Social Science \& Medicine. 1995; 40: 1073-82.

17.- Derluyn I, Broekaert E, Schuyten G: Emotional and behavioural problems inmigrant adolescents in Belgium. Eur Child Adolesc Psychiatry 2008; 17: 54-62.

18.- Urzúa A, Domic M, Cerda A, Ramos M, Quiroz J: Trastorno por Déficit de Atención con Hiperactividad en Niños Escolarizados. Rev Chil Pediatr 2009; 80: 332-8.

19.- Escudero J: Calidad de vida en niños y niñas Aymara escolarizados en la ciudad de Arica. (Tesis de grado). Universidad de Tarapacá, 2011.

20.- Díaz C, Rengifo $S$ : El impacto del proceso de aculturación en la identidad étnica del pueblo Aymara en la XV Región de Arica y Parinacota (Tesis de grado). Universidad de Tarapacá, 2010. 